\title{
Refuerzo externo a cortante en vigas de hormigón mediante aleaciones con memoria de forma en base hierro: de la prueba de concepto a la escala real
}

\section{Shear Strengthening of Reinforced Concrete Beams Through the Use of Iron- Based Shape Memory Alloys: From the Proof-of-Concept to the Real Scale}

\author{
Antoni Cladera ${ }^{a}$, Luis Montoya-Coronado ${ }^{\mathrm{b}}$, Joaquín Ruiz-Pinillac ${ }^{\mathrm{c}}$, Carlos Ribas ${ }^{\mathrm{d}}$ \\ ${ }^{a}$ Catedrático, Dept. de Ingeniería Industrial y Construcción, Universitat de les Illes Balears, Palma (Mallorca), España \\ ${ }^{b}$ Investigador postdoctoral, Dept. de Ingeniería Industrial y Construcción, Universitat de les Illes Balears, Palma (Mallorca), España \\ c Prof. Contratado Doctor, Dept. de Ingeniería Industrial y Construcción, Universitat de les Illes Balears, Palma (Mallorca), España \\ ${ }^{d}$ Prof. Titular, Dept. de Ingeniería Industrial y Construcción, Universitat de les Illes Balears, Palma (Mallorca), España
}

Recibido el 11 de junio de 2019; aceptado el 14 de diciembre de 2020

\section{RESUMEN}

Las barras corrugadas y los flejes de aleación con memoria de forma en base hierro (Fe-SMA, de su denominación en inglés) son ya una realidad en el mercado europeo de la construcción y, de hecho, ya son múltiples los proyectos reales en los que estos elementos se han utilizado para el refuerzo estructural. En este artículo se presentará la tecnología desarrollada por los autores para el refuerzo externo a cortante mediante la utilización de flejes de Fe-SMA a través de dos campañas experimentales: la primera de ellas llevada a cabo como prueba de concepto en vigas de hormigón a pequeña escala, y la segunda en vigas a escala real con sección en T, comentándose las dificultades encontradas y los resultados obtenidos.

2022 Asociación Española de Ingeniería Estructural (ACHE). Publicado por Cinter Divulgación Técnica S.L. Licencia de uso Creative Commons (CC BY-NC-ND 3.0).

PALABRAS CLAVE: Hormigón armado; vigas; refuerzo; cortante; SMA; experimentación.

\section{ABSTRACT}

Iron based shape memory alloy (Fe-SMA) rebars and strips are already a reality in the European construction market and, in fact, there are already many real projects where these elements have been used for strengthening. In this paper, the technology for external shear strengthening using Fe-SMA strips will be presented through two experimental campaigns: the first one carried out as proof-of-concept in small-scale RC beams, and the second one in real-scale beams with T-cross section. The difficulties encountered and the results obtained will be commented.

2022 Asociación Española de Ingeniería Estructural (ACHE). Published by Cinter Divulgación Técnica S.L. Creative Commons License (CC BYNC-ND 3.0).

KEYWORDS: Reinforced concrete; beams; strengthening; shear; SMA; experimentation.

1.

\section{INTRODUCCIÓN}

Las aleaciones con memoria de forma (SMA de sus siglas en inglés) son todavía bastante desconocidas en el sector de la construcción, pese a lo cual se han introducido durante los últimos años en diversos proyectos reales, principalmente en re-

Persona de contacto / Corresponding author:

Correo-e / email: antoni.cladera@uib.es (Antoni Cladera). habilitación $[1,2]$ pero también en algunos proyectos de obra nueva [3], gracias a sus excepcionales propiedades [4]. Entre ellas, destaca su capacidad de alcanzar grandes deformaciones y regresar a una forma definida previamente al descargar o al calentarlas [5], ya sea mediante una aportación directa de calor o mediante la creación de un circuito eléctrico gracias al 
efecto Joule. Estas características diferenciadoras de las SMA hacen de ellas materiales únicos capaces de ser utilizados en estructuras que respondan y se adapten a los cambios del entorno. Sin embargo, la propiedad más característica de estos materiales es que la respuesta al estímul es pronunciada y abrupta, al contrario de lo que pasa con la mayoría de los materiales convencionales, que presentan respuestas proporcionales al estímulo. Además, esta respuesta solo se da para ciertos valores concretos del estímulo, permitiendo largos periodos de inactividad.

La rotura por cortante de un elemento de hormigón es una rotura frágil, que en caso de producirse, podría impedir la creación de roturas plásticas y la rotura del elemento a flexión tras la plastificación de la armadura a flexión. Por este motivo, es frecuente recurrir a refuerzos externos por cortante cuando se evalúa que la resistencia a cortante puede ser crítica, con frecuencia ante el aumento de las cargas o por disminución de la armadura resistente a cortante por problemas de durabilidad. En cualquier caso, la mayor parte de los procedimientos más habituales de refuerzo externo a cortante son de tipo pasivo, es decir, el material de refuerzo externo inicia su colaboración estructural tras un incremento de las cargas aplicadas en la estructura. Este sería el caso, entre otros, de diferentes técnicas de refuerzo con polímeros reforzados con fibras (FRP) $[6,7]$ o, por ejemplo, la utilización externa de flejes de acero o encamisados incluyendo armadura a cortante [8].

En este artículo se presentarán y compararán las características más relevantes del proceso de refuerzo seguido en dos campañas experimentales de refuerzo activo a cortante mediante la utilización de aleaciones con memoria de forma en base hierro, así como los resultados en ambas campañas. La primera de ellas se llevó a cabo a nivel de prueba de concepto, e implicó el ensayo de 10 vigas-probetas de hormigón de pequeño tamaño [9]. La segunda campaña experimental consistió en la realización de 8 ensayos en 4 vigas a escala real [10].

\section{2.}

\section{LAS ALEACIONES CON MEMORIA DE FORMA EN BASE HIERRO}

En la bibliografía técnica se pueden encontrar múltiples estados del conocimiento relativos a la aplicación de las SMA a estructuras de ingeniería civil, entre otros los publicados en las referencias $[4,11,12]$. Por ello, en este apartado solo se mencionarán los aspectos esenciales relacionados con la aleación con memoria de forma utilizada y teniendo en cuenta su aplicación como refuerzo externo a cortante.

La figura 1 presenta de forma esquemática el procedimiento para utilizar las Fe-SMA como pretensado, ya sea longitudinal o transversal, como en el caso del refuerzo a cortante. En primer lugar es necesario producir martensita a nivel atómico, produciendo la transformación martensítica directa, mediante un pre-estiramiento (curva negra en la figura 1.a). Este pre-estiramiento, hasta una deformación de entre el 2 y el $4 \%$, puede haber sido realizado directamente por el proveedor, como es el caso en los flejes utilizados en la segunda campaña experimental descrita en este artículo. Tras la descarga, el material está preparado para su disposi- ción en el elemento estructural, en este caso rodeando la viga, para lo que se debe anclar adecuadamente. Una vez anclado correctamente, es preciso activar el material: esto se consigue incrementando su temperatura para que se produzca la transformación martensítica inversa, transformando la martensita en austenita, por lo que la Fe-SMA querrá recuperar su forma previa al estiramiento, acortándose. Al estar la deformación impedida, se generarán tensiones de recuperación (curva roja en la figura 1). Estas tensiones de recuperación se mantienen tras el posterior enfriamiento. En este punto, el proceso de refuerzo ha finalizado (salvo la posible ejecución de alguna protección ante la corrosión o incendio, por ejemplo mediante hormigón gunitado). Frente a cualquier incremento posterior de carga, el material podrá incrementar su tensión y deformación (curva azul en la figura 1 a).

La curva tensión-deformación del material es no lineal, y esta será diferente en función del estado del material (sin pre-estiramiento, pre-estirado, activado, etc.), por lo que será esencial llevar a cabo la caracterización del material. Se puede consultar más información en $[9,13,14]$.

a)

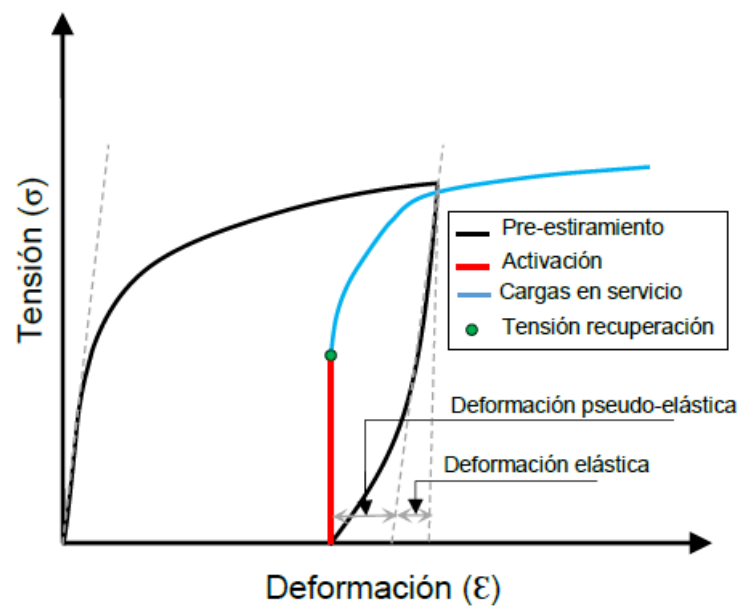

b)

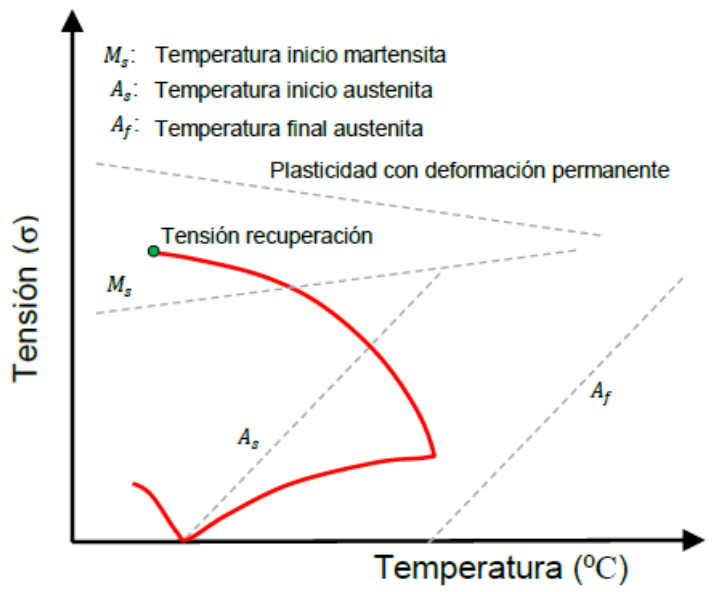

Figura 1. Ilustración del comportamiento de una Fe-SMA.

a) Curva tensión-deformación incluyendo preestiramiento (negro), tensión de recuperación (rojo) y carga (azul).

b) Ilustración de la activación (generación tensiones de recuperación). 
3.

\section{DESCRIPCIÓN DE LAS CAMPAÑAS EXPERIMENTALES}

La tabla 1 presenta las características principales de los 18 ensayos efectuados.

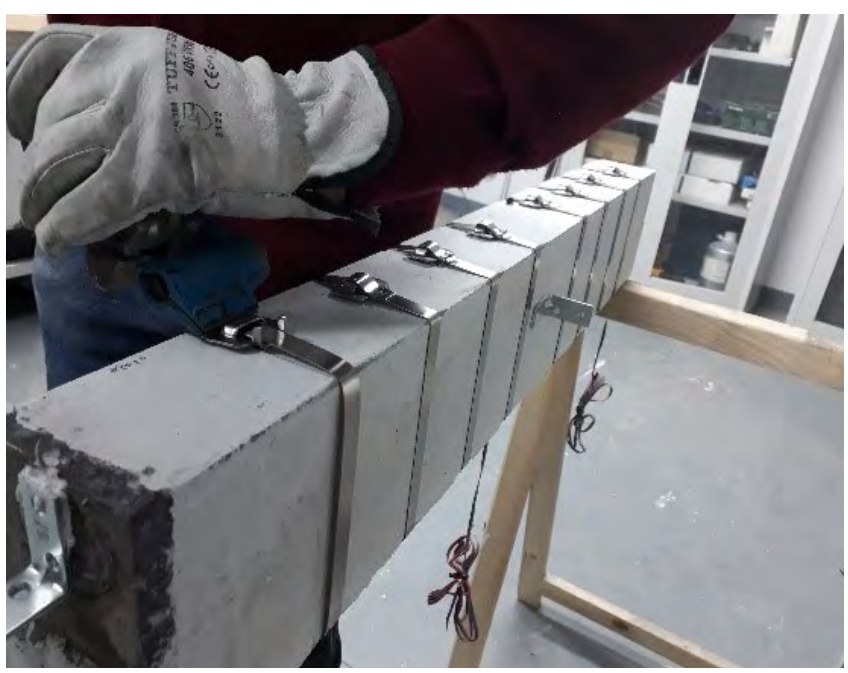

Figura 2. Colocación del refuerzo externo en sección rectangular, utilizando tensor de flejes. Campaña 1.

Las vigas de la campaña experimental 1 eran de pequeña escala (canto de $150 \mathrm{~mm}$; luz a cortante de $340 \mathrm{~mm}$, longitud total $900 \mathrm{~mm}$ ) y de sección rectangular. Por el contrario, las vigas de la campaña experimental 2 se construyeron a escala real (canto de $550 \mathrm{~mm}$; luz a cortante de $1477.5 \mathrm{~mm}$, longitud total $5800 \mathrm{~mm}$ ) y con sección en T. Cabe destacar que el diseño corresponde al previamente utilizado por otros autores en una campaña de refuerzo con láminas de FRP [7].

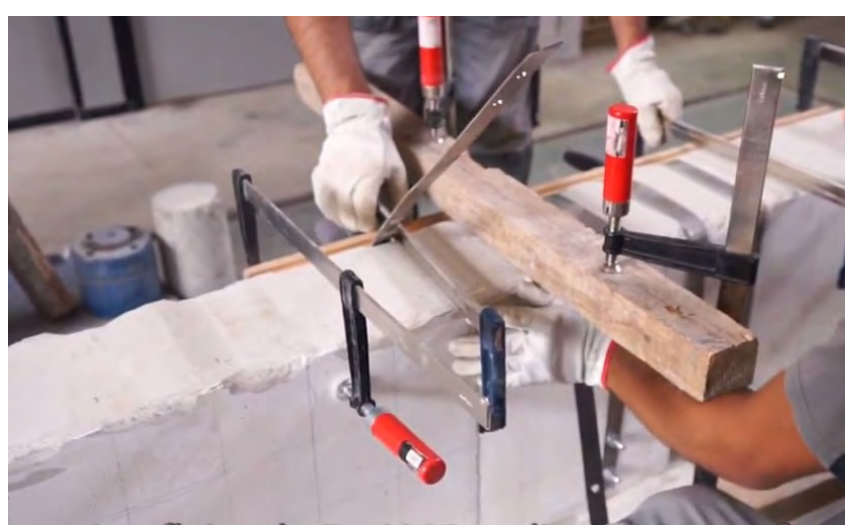

Figura 3. Colocación refuerzo externo. Sección en T.

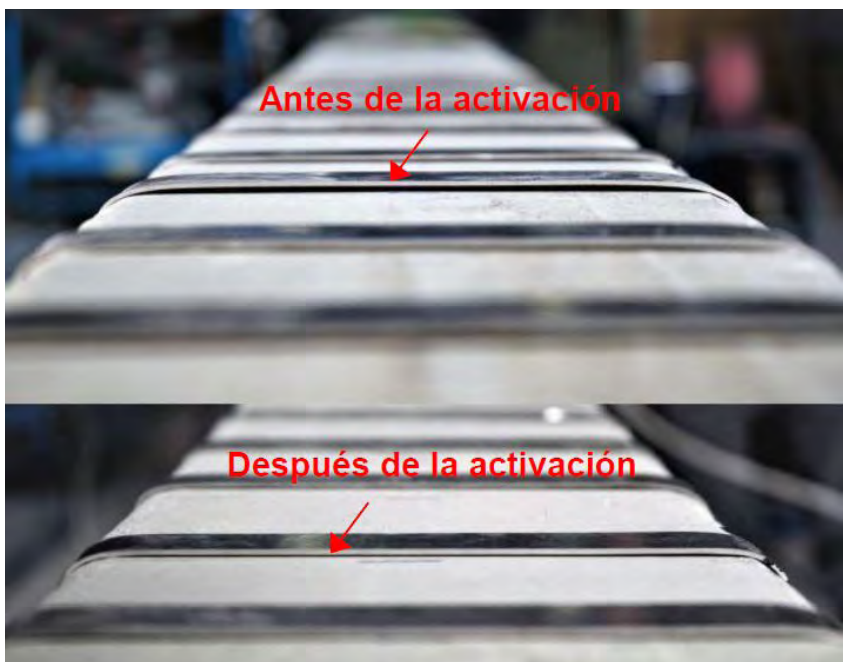

Figura 4. Espacio entre el fleje y el paramento del hormigón antes y después de la activación.

TABLA 1.

Descripción de las campañas experimentales

\begin{tabular}{|c|c|c|c|c|c|c|c|c|c|c|c|}
\hline Campaña & Ensayo & $\begin{array}{c}\mathrm{b} \\
{[\mathrm{mm}]}\end{array}$ & $\begin{array}{c}\mathrm{b}_{\mathrm{w}} \\
{[\mathrm{mm}]}\end{array}$ & $\begin{array}{c}h \\
{[\mathrm{~mm}]}\end{array}$ & $a / d$ & $\begin{array}{c}f_{c m} \\
{[\mathrm{MPa}]}\end{array}$ & $\begin{array}{c}\text { Separación } \\
\text { nominal flejes } \\
{[\mathrm{mm}]}\end{array}$ & $\begin{array}{c}\text { Ancho x } \\
\text { espesor flejes } \\
{[\mathrm{mm}]}\end{array}$ & $\begin{array}{c}\text { Estado } \\
\text { Fe-SMA }\end{array}$ & $\begin{array}{c}V_{\text {test }} \\
{[\mathrm{kN}]}\end{array}$ & $\begin{array}{c}\delta_{\text {test }} a t \\
V_{\text {test }} \\
{[\mathrm{mm}]}\end{array}$ \\
\hline \multirow{10}{*}{$\begin{array}{l}\text { 1: Prueba } \\
\text { de concep- } \\
\text { to }\end{array}$} & $\mathrm{Rl}$ & 80 & 80 & 150 & 2.68 & 30.1 & - & - & - & 17.95 & 3.24 \\
\hline & $\mathrm{R} 2$ & 80 & 80 & 150 & 2.68 & 30.1 & - & - & - & 15.82 & 1.53 \\
\hline & SPl & 80 & 80 & 150 & 2.68 & 30.1 & 90 & $25 \times 0.5$ & No activado & 29.51 & 7.88 \\
\hline & SP2 & 80 & 80 & 150 & 2.68 & 30.1 & 90 & $25 \times 0.5$ & No activado & 31.01 & 9.34 \\
\hline & SAl & 80 & 80 & 150 & 2.68 & 30.1 & 90 & $25 \times 0.5$ & Activado & 31.69 & 6.52 \\
\hline & SA2 & 80 & 80 & 150 & 2.68 & 30.1 & 90 & $25 \times 0.5$ & Activado & 31.64 & 5.83 \\
\hline & SPnl & 80 & 80 & 150 & 2.68 & 30.5 & 90 & $12.5 \times 0.5$ & No activado & 25.18 & 7.05 \\
\hline & SAnl & 80 & 80 & 150 & 2.68 & 30.5 & 90 & $12.5 \times 0.5$ & Activado & 27.55 & 4.14 \\
\hline & Crl-SPnl & 80 & 80 & 150 & 2.68 & 31.0 & 90 & $12.5 \times 0.5$ & No activado & 29.75 & 6.65 \\
\hline & Cr2-SAnl & 80 & 80 & 150 & 2.68 & 31.0 & 90 & $12.5 \times 0.5$ & Activado & 29.15 & 4.69 \\
\hline \multirow[t]{8}{*}{ 2: Escala real } & 1 1-1-R & 600 & 200 & 550 & 3.02 & 33.1 & - & - & - & 227.9 & 15.4 \\
\hline & $1-2-R$ & 600 & 200 & 550 & 3.02 & 33.1 & - & - & - & 299.1 & 18.2 \\
\hline & $2-1-A-5$ & 600 & 200 & 550 & 3.02 & 33.4 & 240 & $60 \times 1.5$ & Activado & 329.3 & 20.4 \\
\hline & $2-2-A-5$ & 600 & 200 & 550 & 3.02 & 33.4 & 240 & $60 \times 1.5$ & Activado & 339.8 & 18.2 \\
\hline & $3-1-A-10$ & 600 & 200 & 550 & 3.02 & 33.5 & 120 & $30 \times 1.5$ & Activado & 363.4 & 20.1 \\
\hline & 3-2-A-10 & 600 & 200 & 550 & 3.02 & 33.5 & 120 & $30 \times 1.5$ & Activado & 353.2 & 21.1 \\
\hline & 4-1-P-10 & 600 & 200 & 550 & 3.02 & 33.5 & 120 & $30 \times 1.5$ & No activado & 358.9 & 19.2 \\
\hline & 4-2-P-10 & 600 & 200 & 550 & 3.02 & 33.5 & 120 & $30 \times 1.5$ & No activado & 329.0 & 18.1 \\
\hline
\end{tabular}


El cambio de tipo de sección obligó a cambiar el procedimiento de montaje. En las pequeñas vigas rectangulares se optó por utilizar flejes que envolvieran completamente la sección transversal (figura 2), resultando en un sistema auto-anclado, pudiéndose utilizar, además, un tensor de flejes manual para minimizar el error de montaje. En cambio, en las vigas con sección en $\mathrm{T}$, se optó por utilizar los flejes disponiéndolos en forma de $\mathrm{U}$, sin atravesar la capa de compresión, y anclando los flejes a cada lado del alma mediante anclajes mecánicos (figura 3). La pequeña separación entre los flejes y el paramento de hormigón existente tras la instalación de los flejes en U (el error de montaje) prácticamente desaparecía durante el proceso de activación, gracias a la recuperación de la forma (figura 4).

Otra diferencia importante entre las vigas de ambas campañas es que los elementos de la campaña 1 (ver tabla 1) no tenían armadura a cortante en su interior, mientras que las vigas de la campaña 2 tenían, en las zonas críticas a cortante, cercos $\varphi 6$ cada $240 \mathrm{~mm}$.

\subsection{Caracterización de los materiales}

Previamente a la realización de los ensayos se llevó a cabo una completa caracterización de los materiales utilizados en ambas campañas experimentales. La información más importante se presenta a continuación.

La resistencia a compresión objetivo en ambas campañas experimentales era de $30 \mathrm{MPa}$, para simular un hormigón no excesivamente resistente de un elemento fabricado con condicionantes menos rígidos que los incluidos en la normativa actual. La resistencia media a compresión para cada viga, estimada para el día del ensayo, se ha presentado en la tabla 1.

En el caso de los elementos de la campaña 1, la armadura longitudinal estaba formada únicamente por 1Ø16. El valor medio del límite elástico de estas barras fue $f_{y}=550 \mathrm{MPa}$ y la resistencia última $f_{u}=649 \mathrm{MPa}$.

En el caso de las vigas de la campaña 2, se utilizaron $4 \varnothing 16$ $+4 \varnothing 20$ como armadura longitudinal. El valor medio del límite elástico fue de $525 \mathrm{MPa}$ y $519 \mathrm{MPa}$, para las barras de ambos diámetros, y la resistencia última, $639 \mathrm{MPa}$ y $623 \mathrm{MPa}$.

Las características de los flejes de Fe-SMA utilizadas en ambas campañas son muy similares, pese a la diferencia de espesor. En la referencia [13] se presenta una caracterización muy detallada de los flejes de espesor $0.5 \mathrm{~mm}$ y de $1.5 \mathrm{~mm}$. A modo de resumen, la figura 5 muestra la curva tensión-deformación del material original y tras la activación de una muestra real de $0.5 \mathrm{~mm}$ (figura 5a), así como la generación real de tensiones de recuperación, en el entorno de $350 \mathrm{MPa}$. El límite elástico, entendido como la tensión para una deformación permanente del $0.2 \%$, fue de $475 \mathrm{MPa}$ y $470 \mathrm{MPa}$ para los flejes de 0.5 y 1.5 $\mathrm{mm}$, respectivamente, y la tensión de rotura 950 y $940 \mathrm{MPa}$

4.

\section{RESULTADOS}

La tabla 1 y la figura 6 resumen los resultados más importantes obtenidos en relación al cortante máximo experimental $\left(V_{\text {test }}\right)$ y la flecha máxima bajo el punto de aplicación de la carga $\left(\delta_{\text {test }}\right)$. Se observa que, en Estado Límite Último, las vigas reforzadas resisten esfuerzos cortantes más elevados en todos los casos, pero con notables diferencias.

En el caso de las vigas de la primera campaña, las vigas reforzadas con flejes de $25 \mathrm{~mm}$ (figura 6a) fallaron a flexión, mientras que las vigas de referencia, o las vigas reforzadas con flejes de $12.5 \mathrm{~mm}$ fallaron a cortante.

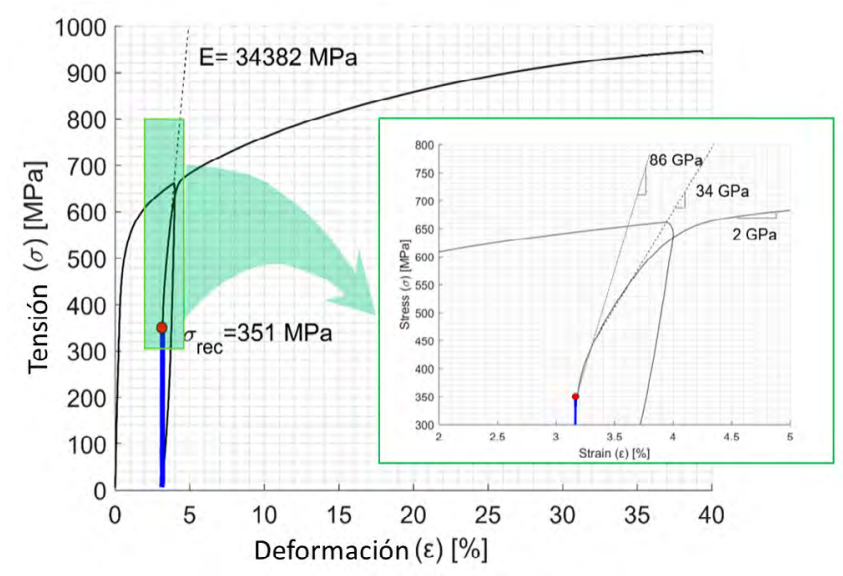

a)

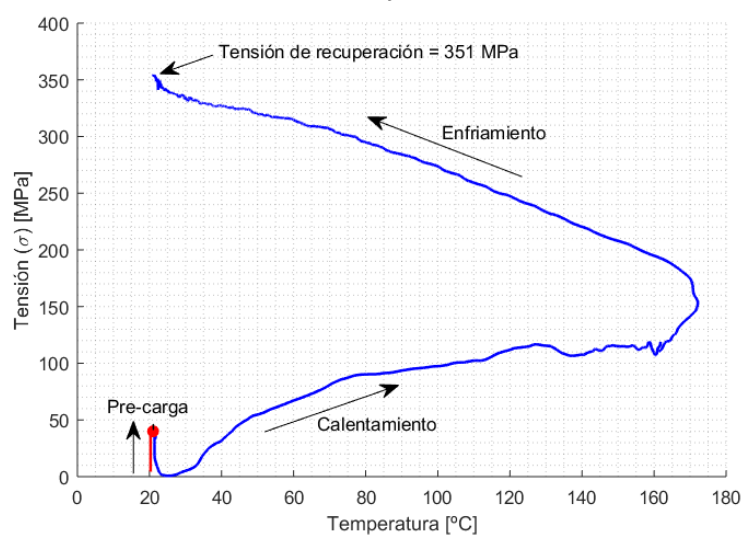

b)

Figura 5.

a) Curva tensión-deformación incluyendo pre-estiramiento, tensión de recuperación (azul) y carga posterior.

b) Generación de tensiones de recuperación (activación) [9].

En la figura $6 \mathrm{~d}$ los ensayos se han agrupado por ensayos equivalentes. En la mayor parte de los casos se tratan de ensayos idénticos, excepto en el caso de las vigas con flejes de 12.5 $\mathrm{mm}$, en que una de las vigas estaba prefisurada ( $\mathrm{Cr} 1$ o $\mathrm{Cr} 2$ ), y su viga gemela no había sido prefisurada. Se observa que en el caso de la primera campaña experimental, el incremento de carga última de las vigas reforzadas está en el entorno de entre el $60 \%$ y algo más del $80 \%$, resistiendo, en valor medio, más las vigas con los flejes activados.

En el caso de las vigas de la segunda campaña (figuras $6 c$ y 6d), el aumento de cortante de rotura se sitúa entre el 27 y $36 \%$, aproximadamente, de lo que resistieron las vigas de referencia. Se trata de un incremento moderado causado, a juicio de los autores, por problemas de anclaje de los flejes en el alma.

En todo caso, la diferencia principal de comportamiento por el efecto de las tensiones de recuperación se debe buscar en la fisuración de las vigas, y por tanto, está relacionada con el 


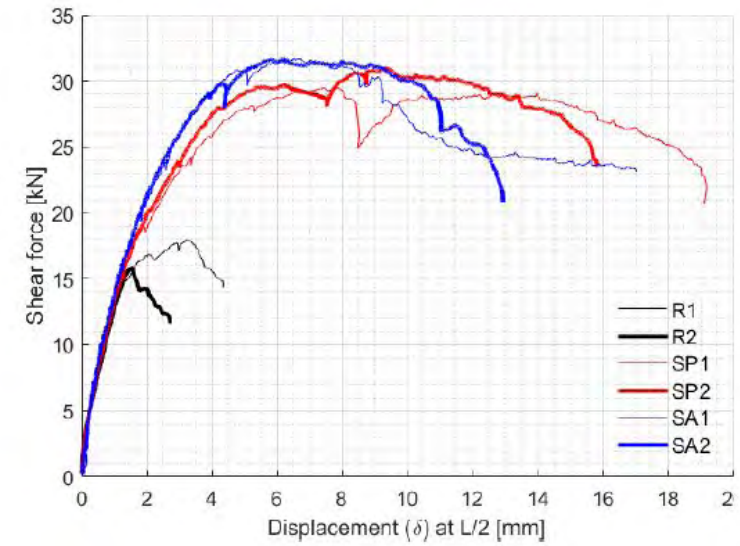

a. Campaña 1: vigas referencia y con flejes de $25 \mathrm{~mm}$.

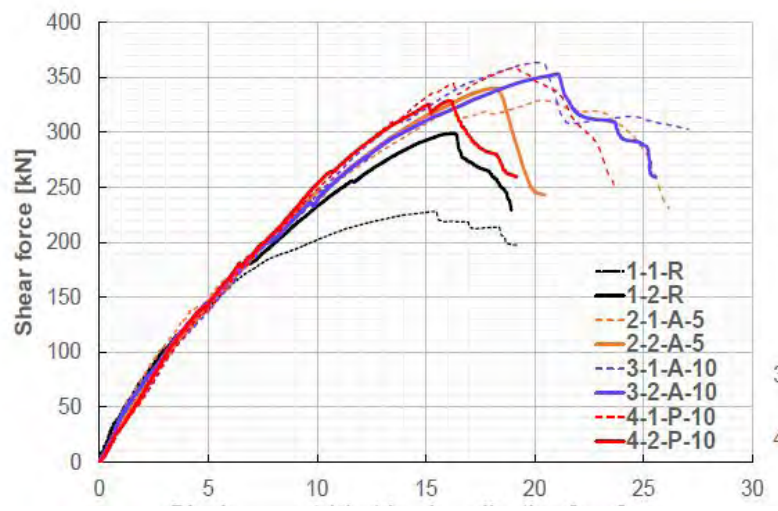

c. Campaña 2: todas las vigas.

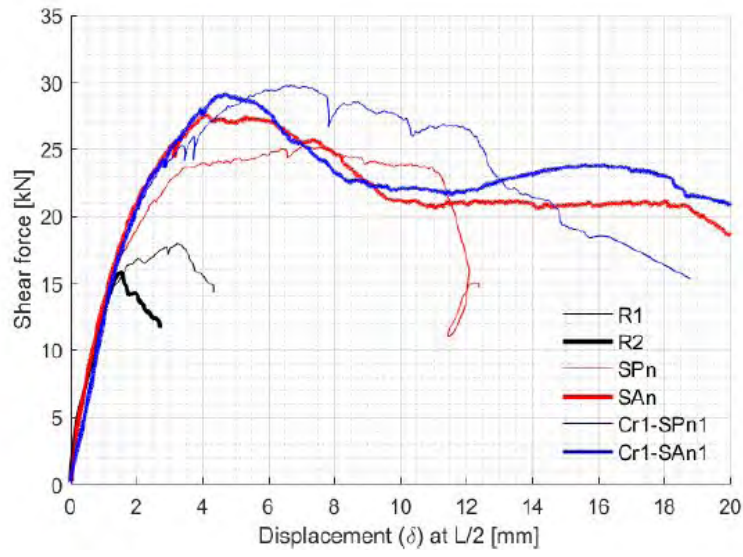

b. Campaña 2: vigas referencia y con flejes de $12.5 \mathrm{~mm}$.

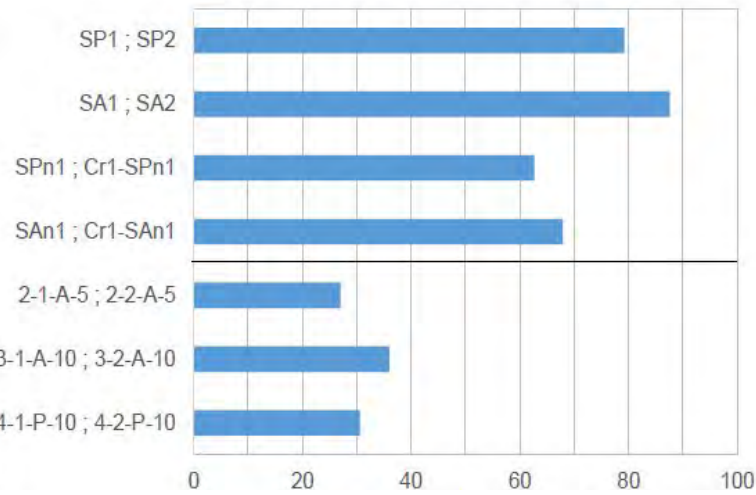

d. Incremento respecto vigas de referencia.

Figura 6. Curvas esfuerzo cortante-flecha e incrementos de carga última a rotura.

estado límite de servicio. La figura 7 presenta, a modo de ejemplo, la fisuración en rotura de dos vigas de la primera campaña experimental. Las vigas son idénticas, pero en la representada en la figura 7a (SP1) los flejes se comportan de forma pasiva, al no haber sido previamente activados. En contra, en la viga de la figura $7 \mathrm{~b}$ (SA1), los flejes han sido activados antes de la aplicación de la carga. Se observa claramente que las fisuras a cortante que aparece en ambos vanos de la viga SPl, entre el soporte y el punto de aplicación de la carga, no se ha producido en el caso de la viga SAl con flejes activos.

En el caso de todas las demás vigas que fallaron por cortante de la primera campaña experimental, se observó un retraso en la aparición de la fisuración por cortante para las vigas con flejes activados.

\section{a)}

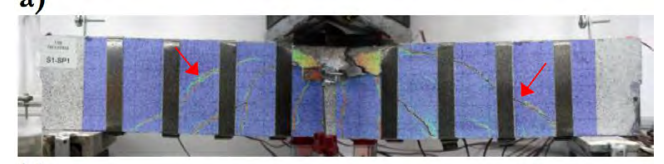

b)

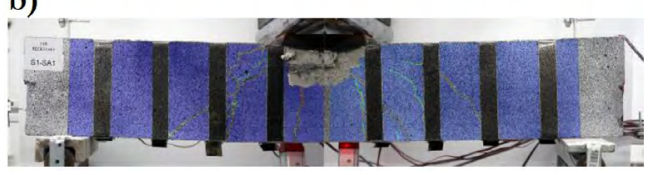

Figura 7. Fisuración de las vigas justo después de la carga máxima. a) Viga SP1: flejes de $25 \mathrm{~mm}$ sin activación.

b) Viga SAl: flejes de $25 \mathrm{~mm}$ activados.
TABLA 2.

Cortante para abertura de fisura de $0.3 \mathrm{~mm}$ en campaña experimental 2.

\begin{tabular}{lccc}
\hline Ensayo & $\begin{array}{c}\text { Media } \\
\mathbf{V}_{\omega=0.3 \mathrm{~mm}} \\
{[\mathrm{kN}]}\end{array}$ & $\begin{array}{c}\mathbf{V}_{\omega=0.3 \mathrm{~mm}} \\
{[\mathrm{kN}]}\end{array}$ & $\begin{array}{c}\Delta \mathbf{V}_{\omega=0.3 \mathrm{~mm}} \\
{[\%]}\end{array}$ \\
\hline $1-1-\mathrm{R}$ & 123 & 123 & - \\
1-2-R & 123 & & 33 \\
\hline 2-1-A-5 & 140 & 164 & 24 \\
2-2-A-5 & 187 & & \\
\hline 3-1-A-10 & 142 & 152 & 12 \\
3-2-A-10 & 162 & & \\
\hline 4-1-P-10 & 138 & 138 & \\
$4-2-\mathrm{P}-10$ & 139 & & \\
\hline
\end{tabular}

En la campaña experimental 2 se midió, mediante la utilización de transductores de desplazamiento tipo Encoder, la deformación diagonal a tracción y compresión en la zona de la fisura crítica del alma. Si bien no se trata de una medida exacta, la medida del transductor a tracción puede asimilarse a la apertura de fisura. A partir de estas lecturas, en la tabla 2 se presenta el valor del cortante para el que la deformación por tracción en el alma es igual a $0.3 \mathrm{~mm}$. Se observa que el valor medio del cortante se incrementa entre un 24 y un 33\% para el caso de vigas con flejes activados, mientras que el incremento 
es más modesto (12\%) en el caso de flejes sin activar (refuerzo pasivo).

\section{5. \\ DISCUSIÓN}

El refuerzo activo exterior a cortante mediante la utilización de flejes de Fe-SMA es una tecnología muy novedosa de la que se disponen, a día de hoy, muy pocos resultados publicados en revistas científicas, por este motivo, cualquier discusión debe ser entendida con cautela.

En las dos campañas experimentales llevadas a cabo, se ha mostrado un incremento del esfuerzo de rotura por cortante y un mejor comportamiento en cuanto a la aparición de fisuras, lo que coincide con los resultados muy recientemente publicados sobre una campaña experimental que utiliza barras corrugadas de la misma aleación para el refuerzo externo a cortante [15]. Sin embargo, el resultado para las vigas a escala real no ha sido tan satisfactorio como se esperaba.

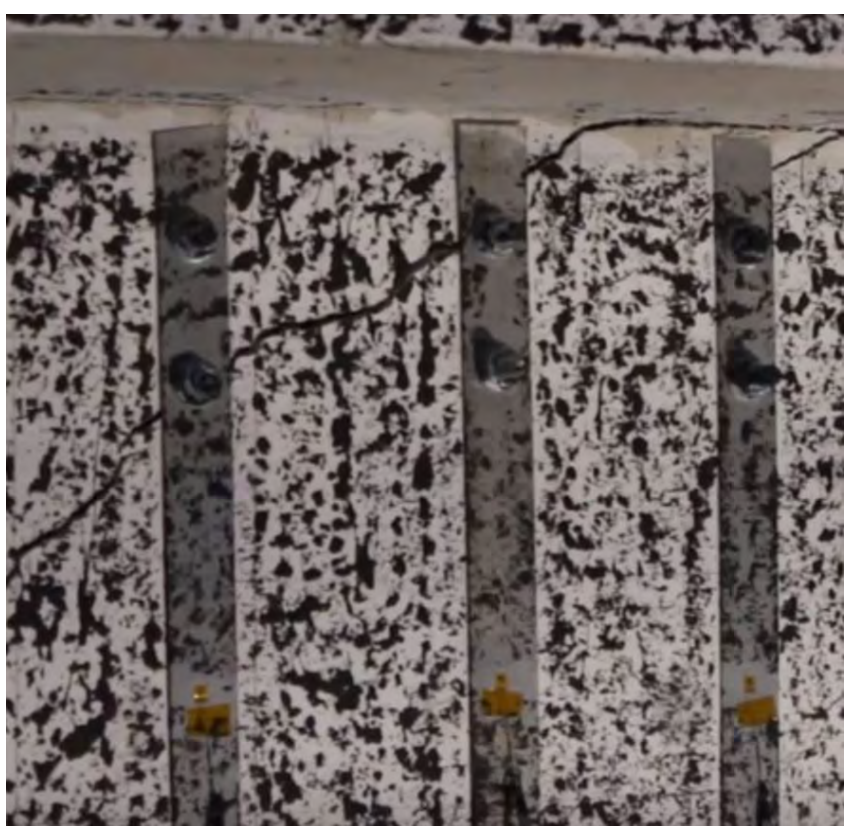

Figura 8. Detalle de fisura que se propaga produciendo el fallo de los anclajes mecánicos en el alma.

A falta de un mayor número de resultados experimentales, la diferencia de nivel de refuerzo obtenido entre las dos campañas experimentales podría deberse más al cambio de la sección transversal del elemento, de sección rectangular a sección en $\mathrm{T}$, lo que implicó un cambio en el sistema de anclaje, que a algún tipo de fenómeno relacionado con la escala.

$\mathrm{Al}$ inicio de la campaña experimental se tomó la decisión de reforzar las vigas intentando evitar la perforación del ala comprimida, lo que obligó a utilizar refuerzos externos en forma de $\mathrm{U}$, anclados en el alma, tal y como se ha propuesto en muchísimos trabajos para refuerzos externos con FRP. Sin embargo, tras el transcurso de los ensayos se llegó a la conclusión de que la decisión no fue probablemente la óptima por dos motivos:

- Los anclajes mecánicos se fijaban en el alma, sometida a tracciones diagonales, debilitándola en cierto modo. De esta forma, las fisuras a cortante invariablemente se dirigían a las fijaciones mecánicas, tal y como se aprecia en la figura 8, produciendo el fallo de los anclajes mecánicos y, por tanto, impidiendo el trabajo correcto de los flejes.

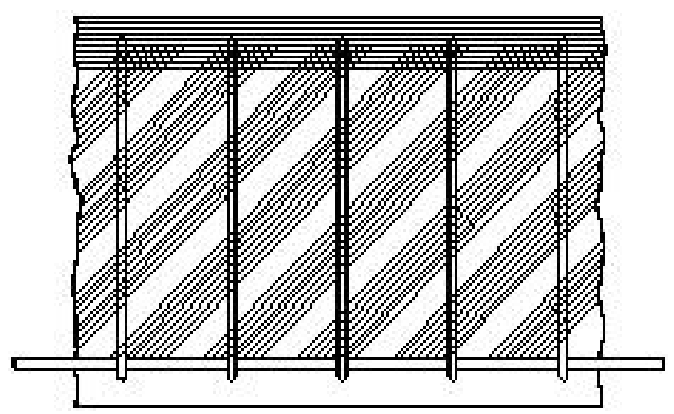

Figura 9. Modelo de celosía de Mörsch.

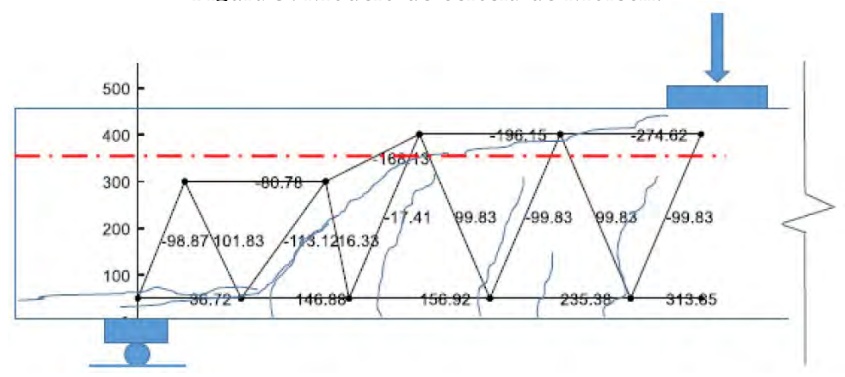

Figura 10. Modelo de celosía optimizado considerando la fisuración real de un ensayo [16].

- En cualquier modelo de resistencia a cortante, desde el antiguo modelo de Mörsch representado en la figura 9, hasta un modelo de celosía optimizado considerando la fisuración real durante un ensayo, figura 10, se necesita que la tracción del alma se transmita desde la armadura longitudinal a tracción al cordón comprimido (que podría tener altura variable), siempre y cuando el cortante no se resista exclusivamente mediante el efecto arco. En cualquier caso, el efecto arco se puede obviar, en general, para relaciones a/d mayores a 2.5, excepto en la zona más próxima al apoyo (zona de discontinuidad), tal y como se observa en la figura 10. El hecho de que los flejes externos se anclasen por debajo de la cabeza comprimida impidió el correcto "cierre" de la celosía a cortante, por lo que la resistencia de la viga estaba condicionada por la resistencia de la zona superior del alma, por encima del anclaje y por debajo del cordón comprimido.

La combinación de los dos fenómenos anteriores podría haber afectado de forma significativa a la resistencia de las vigas ensayadas, por lo que se propone como futuros trabajos, el ensayo de vigas con sección en $\mathrm{T}$ utilizando flejes que envuelvan el alma de la viga, perforando la cabeza comprimida.

\section{6. \\ MODELIZACIÓN NUMÉRICA}

\subsection{Curva tensión-deformación}

Tal y como se ha representado de forma esquemática en la figura 1, una vez el refuerzo externo ha sido instalado y acti- 
vado, la curva tensión-deformación tendrá un carácter fuertemente no lineal para valores crecientes de carga (curva azul en la figura 1). En el origen de la curva tensión-deformación, un valor nulo de deformaciones relativas corresponde a la llamada tensión de recuperación, como se aprecia en la figura 11 en el eje de referencia principal $\sigma-\varepsilon$. Este comportamiento puede ser modelado a través de la adaptación de la formulación de Ramberg-Osgood y sus extensiones [17-20]. La curva presentada en la figura 11 ha sido la utilizada para llevar a cabo el modelo de elementos finitos de las vigas. Nótese que la curva se define en dos tramos, en función del preestiramiento inicial llevado a cabo para generar la martensita (curva en negro en la figura 1, y en gris claro en la figura 11). En la referencia [14] se presentan detalladamente las ecuaciones.

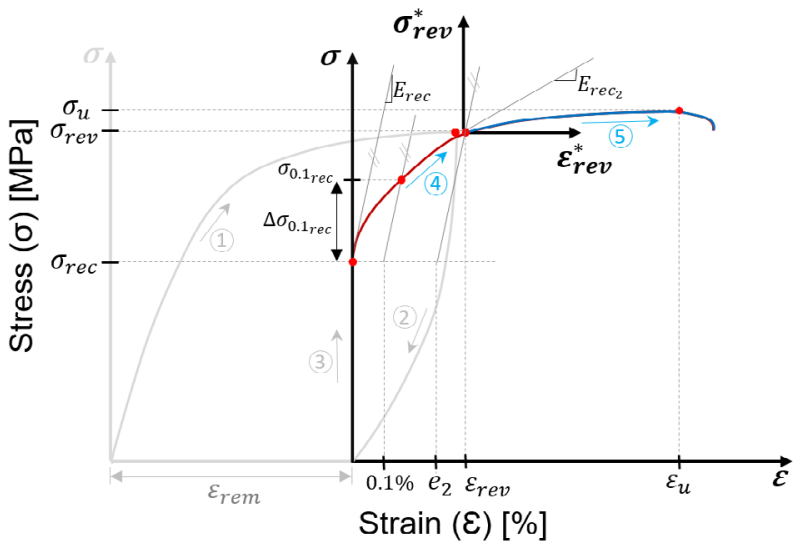

Figura 11. Curva de comportamiento tensión-deformación del Fe-SMA tras la activación.

\subsection{Modelo de elementos finitos}

Se ha realizado la modelización numérica de las vigas mediante elementos finitos en ATENA 2D [21]. Los modelos consideran las leyes constitutivas no lineales del hormigón, del armado y del Fe-SMA tanto activado como no activado propuestas en [14]. El software combina modelos de tracción [22] y de compresión [23] dependiendo del daño progresivo y las deformaciones plásticas alcanzadas, modelizando la fractura mediante fisuras distribuidas en bandas [24]. Además, ha tenido en cuenta las condiciones de adherencia entre el hormigón y el armado interior según [25]. Para el armado pasivo se ha utilizado una ley bilineal con endurecimiento, y para el Fe-SMA se han usado las curvas representadas en la figura 11. Las vigas no reforzadas se han considerado como vigas de referencia para la calibración de los parámetros del hormigón armado, variando el tamaño de la malla y los parámetros de plasticidad hasta obtener una aproximación razonable con la experimentación. Puede consultarse más información en [14] para el caso de las vigas de la primera campaña experimental.

En la campaña experimental se realizaron dos ensayos para cada tipo de viga, de modo que para cada modelo numérico hay dos ensayos experimentales con los que contrastar los resultados. En la tabla 3 se muestran los resultados medios obtenidos, que presentan una buena aproximación. Destaca que los resultados con más dispersión se dan en las vigas de referencia, mientras que para las vigas reforzadas ex- ternamente externamente, el error es siempre inferior al 9\%. Además, los patrones de fisuración de las vigas ensayadas y los predichos mediante el modelo numérico son muy similares, como se presenta en la figura 12 para algunas vigas seleccionadas.

TABLA 3.

Comparación entre resistencias a cortante experimentales y obtenidas por el modelo numérico.

\begin{tabular}{cccc}
\hline \multicolumn{5}{c}{ Máximo cortante soportado [kN] } \\
\hline & Experimental & MEF & MEF/EXP \\
\hline 1. Vigas sección rectangular & & & \\
\hline R1 & 17.95 & 15.45 & 0.86 \\
R2 & 15.82 & & 0.98 \\
\hline SP1 & 29.51 & 31.92 & 1.08 \\
SP2 & 31.01 & & 1.03 \\
\hline SAl & 31.69 & 32.91 & 1.04 \\
SA2 & 31.64 & & 1.04 \\
\hline 2. Vigas sección en T & & & \\
\hline 1-1-R & 227.91 & 276.60 & 1.21 \\
1-2-R & 229.10 & & 0.92 \\
\hline 3-1-A & 363.38 & 357.20 & 0.98 \\
3-2-A & 353.21 & & 1.01 \\
\hline 4-1-P & 358.89 & 360.20 & 1.00 \\
4-2-P & 328.96 & & 1.09 \\
\hline & & Promedio & 1.02 \\
& & Desviación estándar & 0.09 \\
\hline
\end{tabular}

En la figura 12d se aprecia también la elevada concentración de daño en la zona comprendida entre la zona del anclaje de los flejes (estos se representan como líneas de trazo discontinuo vertical en la figura del modelo numérico) y las alas, tal y como se había discutido en la sección 5 fruto de las observaciones llevadas a cabo en el laboratorio.

\section{7.}

\section{CONCLUSIONES}

En este artículo se han presentado dos campañas experimentales de refuerzo activo a cortante en vigas de hormigón armado, la primera de ellas a pequeña escala y la segunda a escala real.

Al tratarse de una tecnología muy novedosa, es necesario ser cauteloso con las conclusiones y es preciso continuar con el trabajo de investigación. En cualquier caso, en ambas campañas se ha obervado un mejor comportamiento en condiciones de servicio y un aumento de la carga última resistida. Sin embargo, el comportamiento de las vigas a escala real y con sección en $\mathrm{T}$, reforzadas con Fe-SMA, ha sido menos satisfactorio que el de las vigas a pequeña escala con sección rectangular. A falta de ratificación experimental, el motivo puede ser explicado por el refuerzo en forma de $U$ utilizado en el caso de las vigas con sección en T, que no "cerraba" la celosía a cortante, y que no conseguía un perfecto anclaje de la armadura de refuerzo al dirigirse las fisuras de cortante a la ubicación de los anclajes. Se recomienda disponer, en el futuro, los anclajes mecánicos 

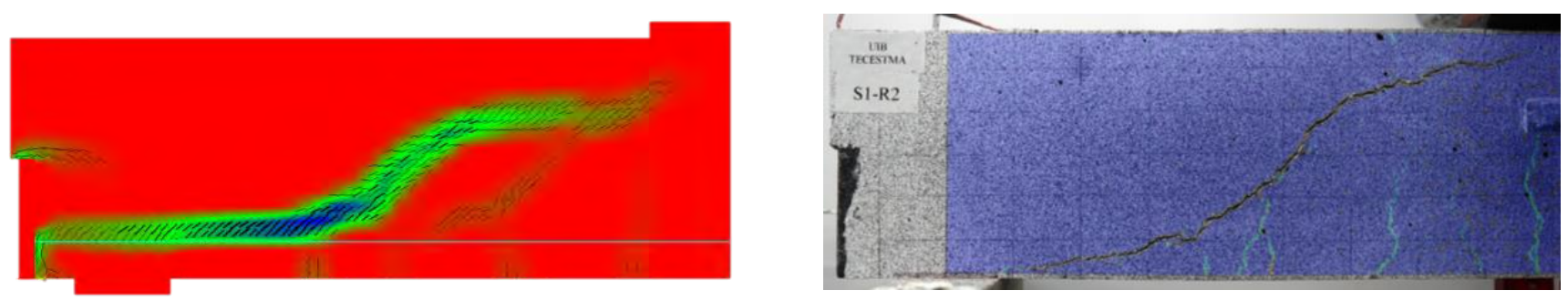

a) Viga de referencia campaña $1:$ R2
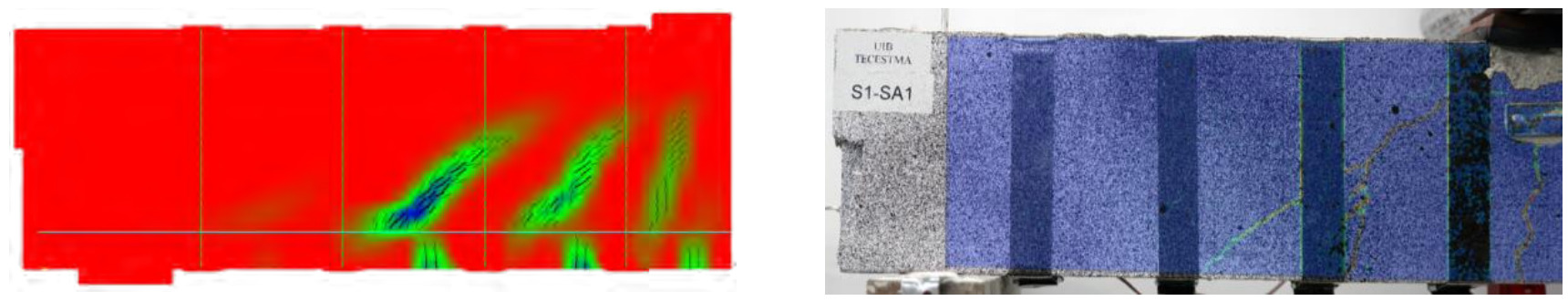

b) Viga campaña 1 con flejes activados: SAl
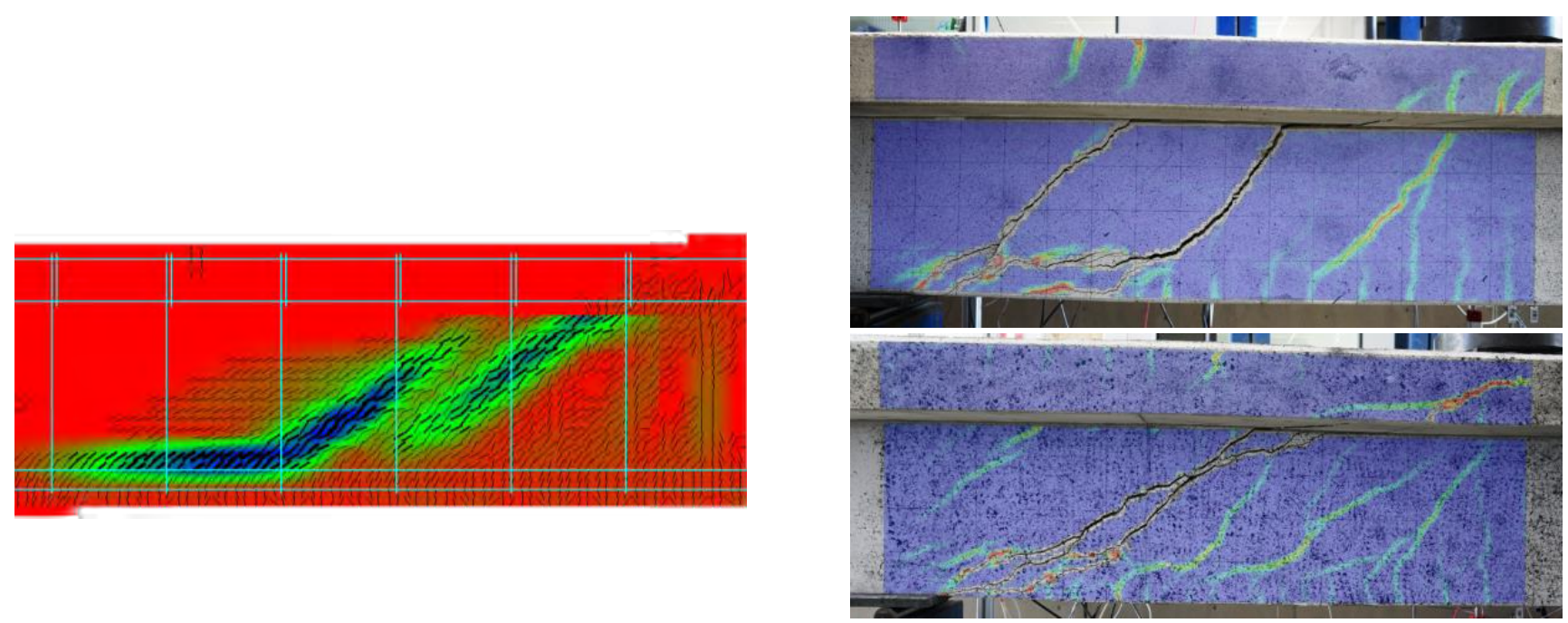

c) Vigas de referencia campaña 2: 1-1-R (superior) y 1-2-R (inferior)
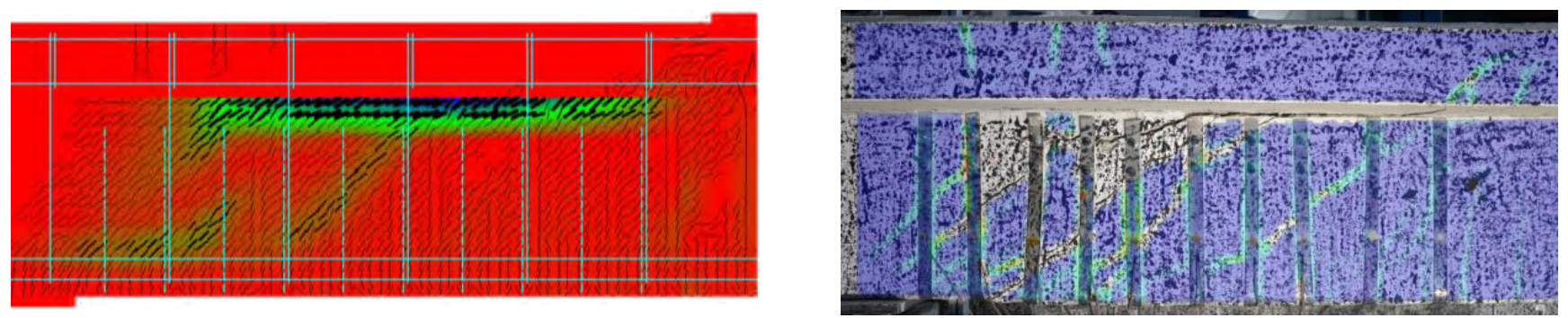

d) Viga campaña 2 con flejes activados: 3-1-A

Figura 12. Patrón de fisuración de vigas de sección rectangular. Los colores representan las deformaciones máximas en el hormigón. A la izquierda los modelos numéricos. A la derecha DIC.

de los flejes en la parte superior de la cabeza comprimida, de forma que el refuerzo envuelva totalmente el alma de la viga, como es el caso de la primera campaña experimental presentada, evitando la concentración del daño en la zona superior del alma, como se observó durante los ensayos y a través del modelo de elementos finitos presentado.

\section{Agradecimientos}

Esta investigación ha sido posible gracias a tres proyectos de investigación. Los ensayos a escala real se han realizado con la Beca Leonardo a Investigadores y Creadores Culturales 2018 de la Fundación BBVA. La Fundación no se responsabiliza de 
las opiniones, comentarios y contenidos incluidos en el proyecto y/o los resultados obtenidos del mismo, los cuales son total y absoluta responsabilidad de sus autores. Los ensayos a pequeña escala se realizaron en el marco del proyecto Horvital -sp2 (BIA2015-64672-C4-3-R) cofinanciado por la Agencia Estatal de Investigación y Fondos FEDER (UE), mientras que el análisis de resultados conjuntos forma parte del proyecto RTI2018099091-B-C22 (AEI / FEDER, UE). Los autores, además, desean agradecer a re-fer AG Strengthening Solution Company por el suministro de los flejes de SMA y a la empresa Labartec, y en especial a Victoriano García, por habernos permitir ejecutar los ensayos a escala real en su laboratorio, gracias a un convenio con la UIB. Los flejes fueron cortados mediante chorro de agua por Raúl Sánchez Torres, técnico de los servicios científico-técnicos de la UIB, al que queremos agradecer su contribución.

\section{Referencias}

[1] Schranz, B., Michels, J., Shahverdi, M., Czaderski, C. (2019) Strengthening of concrete structures with iron-based shape memory alloy elements: Case studies, in: SMAR 2019 - Fifth Conf. Smart Monit. Assess. Rehabil. Civ. Struct., Zurich, Switzerland.

[2] Mercier, J., Basile, B., Hallopeau, X., Michels, J., Tourneur, C. (2019) Structural concrete strengthening with Fe-SMA strips: case study with specific control after activation, in: SMAR 2019 - Fifth Conf. Smart Monit. Assess. Rehabil. Civ. Struct., Zurich, Switzerland.

[3] Varela, S., Saiid Saiidi, M. (2016) A bridge column with superelastic $\mathrm{NiTi}$ SMA and replaceable rubber hinge for earthquake damage mitigation, Smart Mater. Struct. 25 (2016) 075012. doi:10.1088/09641726/25/7/075012

[4] Mas, B., Cladera, A., Ribas, C. (2016) Fundamentos y aplicaciones piloto de las aleaciones con memoria de forma para su utilización en ingeniería estructural, Hormigón y Acero. 67 (2016) 309-323. doi:10.1016/j. hya.2016.02.007.

[5] Otsuka, K., Wayman, C.M. (1998) Shape Memory Materials, Cambridge University Press, United Kingdom.

[6] Foster, R.M., Brindley, M., Lees, J.M., Ibell, T.J., Morley, C.T., Darby, A.P., Evernden, M.C. (2017) Experimental Investigation of Reinforced Concrete T-Beams Strengthened in Shear with Externally Bonded CFRP Sheets, J. Compos. Constr. 21 04016086. doi:10.1061/(ASCE) CC.19435614.0000743

[7] Mari, A., Oller, E., Pujol, M. (2019) Contribution of externally bonded FRP shear reinforcement to the shear strength of RC beams, Compos. Part B Eng. 164 (2019) 235-248. doi:10.1016/j.compositesb.2018.11.065.

[8] Adhikary, B.B., Mutsuyoshi, H. (2006) Shear strengthening of reinforced concrete beams using various techniques, Constr. Build. Mater. 20 (2006) 366-373. doi:10.1016/j.conbuildmat.2005.01.024.
[9] Montoya-Coronado, L.A., Ruiz-Pinilla, J.G., Ribas, C., Cladera, A. (2019) Experimental study on shear strengthening of shear critical RC beams using iron-based shape memory alloy strips, Eng. Struct. 200 (2019) 109680. doi:10.1016/j.engstruct.2019.109680.

[10] Cladera, A., Montoya-Coronado, L.A., Ruiz-Pinilla, J.G., Ribas, C. (2020) Shear strengthening of slender reinforced concrete T-shaped beams using iron-based shape memory alloy strips, Eng. Struct. 221 (2020). doi:10.1016/j.engstruct.2020.111018.

[11] Janke, L., Czaderski, C., Motavalli, M., Ruth, J. (2005) Applications of shape memory alloys in civil engineering structures - Overview, limits and new ideas, Mater. Struct. Constr. 38 (2005) 578-592. doi:10.1617/14323.

[12] Cladera, A., Weber, B.. Leinenbach, C., Czaderski, C., Shahverdi, M., Motavalli, M. (2014) Iron-based shape memory alloys for civil engineering structures: An overview, Constr. Build. Mater. 63 (2014) 281-293. doi:10.1016/j.conbuildmat.2014.04.032.

[13] Shahverdi, M., Michels, J., Czaderski, C., Motavalli, M. (2018) Iron-based shape memory alloy strips for strengthening RC members: Material behavior and characterization, Constr. Build. Mater. 173 (2018) 586-599. doi:10.1016/j.conbuildmat.2018.04.057.

[14] Ruiz-Pinilla, J.G., Montoya-Coronado, L.A., Ribas, C., Cladera, A. (2020) Finite element modeling of RC beams externally strengthened with iron-based shape memory alloy (Fe-SMA) strips, including analytical stress-strain curves for Fe-SMA, Eng. Struct. 223 (2020). doi:10.1016/j. engstruct.2020.111152.

[15] Czaderski, C., Shahverdi, M., Michels, J. (2020) Iron based shape memory alloys as shear reinforcement for bridge girders, Constr. Build. Mater. (2020) 121793. doi:10.1016/j.conbuildmat.2020.121793.

[16] Bairán, J.M., Marí, A., Cladera, A. (2018) Analysis of shear resisting actions by means of optimization of strut and tie models taking into account crack patterns, Hormigón y Acero. 69 (2018) 197-206. doi:10.1016/j. hya.2017.04.009.

[17] Ramberg W.; Osgood W, (1943) Description of stress-strain curves by three parameters, Tech. Note No. 902. Washington, D.C., USA Natl. Advis. Comm. Aeronaut.

[18] Rasmussen, K.J.R.(2003) Full-range stress-strain curves for stainless steel alloys, J. Constr. Steel Res. 59 (2003) 47-61.

[19] Mirambell, E. (2000) Real, On the calculation of deflections in structural stainless steel beams: An experimental and numerical investigation, J. Constr. Steel Res. 54 (2000) 109-133. doi:10.1016/S0143974X(99)00051-6.

[20] Arrayago, I., Real, E., Gardner, L. (2015) Description of stress-strain curves for stainless steel alloys, Mater. Des. 87 (2015) 540-552. doi:10.1016/j. matdes.2015.08.001.

[21] Červenka, V., Jendele, L., Prague, J.Č. (2018) ATENA Program Documentation Part 1 Theory Written by,.

[22] Hordijk, D.A. (1991) Thesis - Local approach to fatigue of concrete.

[23] Menetrey, Ph, Willianm, K.J. (1995) Triaxial Failure Criterion for Concrete and its Generalization, ACI Struct. J. 92 (1995) 311-318. doi:10.14359/1132.

[24] Bažant, Z.P., Oh B.H. (1983) Crack band theory for fracture of concrete, Matériaux Constr. 16 (1983) 155-177. doi:10.1007/BF02486267.

[25] Jendele, L., Cervenka, J. (2006) Finite element modelling of reinforcement with bond, Comput. Struct. 84 (2006) 1780-1791. doi:10.1016/j. compstruc.2006.04.010. 